Claremont Colleges

Scholarship@ Claremont

Pomona Faculty Publications and Research

Pomona Faculty Scholarship

$1-1-2002$

\title{
Asymmetric Two-Colourings of Graphs in $\mathrm{S}^{3}$
}

Erica Flapan

Pomona College

David Linnan Li

Stanford University

\section{Recommended Citation}

ERICA FLAPAN and DAVID LINNAN LI (2002). Asymmetric two-colourings of graphs in S3. Mathematical Proceedings of the Cambridge Philosophical Society, 132, pp 267-280. doi:10.1017/S0305004101005643.

This Article is brought to you for free and open access by the Pomona Faculty Scholarship at Scholarship @ Claremont. It has been accepted for inclusion in Pomona Faculty Publications and Research by an authorized administrator of Scholarship @ Claremont. For more information, please contact scholarship@cuc.claremont.edu. 
Mathematical Proceedings of the Cambridge Philosophical Society

http://journals.cambridge.org/PSP

Additional services for Mathematical Proceedings of the Cambridge

Philosophical Society:

Email alerts: $\underline{\text { Click here }}$

Subscriptions: $\underline{\text { Click here }}$

Commercial reprints: $\underline{\text { Click here }}$

Terms of use : $\underline{\text { Click here }}$

\section{Asymmetric two-colourings of graphs in $\mathrm{S}^{3}$}

ERICA FLAPAN and DAVID LINNAN LI

Mathematical Proceedings of the Cambridge Philosophical Society / Volume 132 / Issue 02 / March 2002 , pp 267 - 280

DOI: 10.1017/S0305004101005643, Published online: 14 March 2002

Link to this article: http://journals.cambridge.org/abstract S0305004101005643

How to cite this article:

ERICA FLAPAN and DAVID LINNAN LI (2002). Asymmetric two-colourings of graphs in $\mathrm{S}^{3}$. Mathematical Proceedings of the Cambridge Philosophical Society, 132, pp 267-280 doi:10.1017/S0305004101005643

Request Permissions : $\underline{\text { Click here }}$ 


\title{
Asymmetric two-colourings of graphs in $S^{3}$
}

\author{
BY ERICA FLAPAN \\ Department of Mathematics, Pomona College, Claremont, CA 91711, U.S.A. \\ e-mail: eflapan@pomona.edu \\ AND DAVID LINNAN LI \\ Department of Mathematics, Stanford University, Stanford, CA 94305, U.S.A. \\ e-mail: daveli@leland.stanford.edu
}

(Received 6 September 1999; revised 12 February 2001)

\section{Abstract}

We prove that for any non-planar graph $H$, we can choose a two-colouring $G$ of $H$ such that $G$ is intrinsically chiral, and if $H$ is 3 -connected and is not $K_{3,3}$ or $K_{5}$, then $G$ is intrinsically asymmetric. No such asymmetric two-colouring is possible for $K_{3,3}$ or $K_{5}$.

The study of graphs embedded in $S^{3}$ is an extension of knot theory which is also related to chemistry. In particular, a molecule can be modelled as a graph in $R^{3}$ where the vertices represent atoms and the edges represent bonds. For such a molecular graph, different coloured edges can be used to represent molecular chains of different types. Symmetries are important in analysing embedded graphs, just as they are in analysing knots. From a chemical point of view, symmetries are significant because they play a role in determining the chemical properties of the molecule. Colouring the edges of a graph with two distinct colours can have the effect of eliminating some of the symmetries of the graph. In this paper, we will discuss how to two-colour most non-planar graphs so that none of their embeddings has any symmetries.

We begin with some definitions. A graph $G$ is a collection of vertices $V$ and edges $E$, such that there is at most one edge between a pair of vertices, and every edge has two distinct vertices. An automorphism of $G$ is a permutation of the vertices of $G$ which preserves adjacency. Suppose that $f$ is an automorphism of a graph $G$, and suppose that $v_{1}$ and $w_{1}$ are adjacent vertices. If $f\left(v_{1}\right)=v_{2}$ and $f\left(w_{1}\right)=w_{2}$ we shall abuse notation slightly and write $f\left(\overline{v_{1} w_{1}}\right)=\overline{v_{2} w_{2}}$. A two-coloured graph $G$ is a graph where each edge of $G$ is either coloured black or white. An automorphism $f$ of a two-coloured graph preserves coloured adjacency. That is, if $v$ and $w$ are connected by a black edge, then $f(v)$ and $f(w)$ are connected by a black edge, and if $v$ and $w$ are connected by a white edge, then $f(v)$ and $f(w)$ are connected by a white edge. Suppose $G$ is a graph which is embedded in $S^{3}$. We define a graph homeomorphism $h:\left(S^{3}, G\right) \rightarrow\left(S^{3}, G\right)$ to be a homeomorphism of $S^{3}$ such that $h(G)=G, h(V)=V$ and $h(E)=E$. If $G$ is a two-coloured graph then a graph homeomorphism of $\left(S^{3}, G\right)$ is also required to be colour preserving. We are interested in which automorphisms of 
a graph are induced by a homeomorphism of $S^{3}$. Some graphs have automorphisms which cannot be induced by a homeomorphism of $S^{3}$ no matter how the graph is embedded [2]. A graph $G$ which is embedded in $S^{3}$ is said to be asymmetric if no non-trivial automorphism of $G$ can be induced by a graph homeomorphism of $\left(S^{3}, G\right)$. An embedded graph $G$ is said to be topologically achiral if there exists an orientation reversing graph homeomorphism $h:\left(S^{3}, G\right) \rightarrow\left(S^{3}, G\right)$. If no such graph homeomorphism exists, then we say that this embedding of $G$ is topologically chiral. If all embeddings of $G$ in $S^{3}$ are topologically chiral, then we say $G$ is intrinsically chiral; and if all embeddings of $G$ in $S^{3}$ are asymmetric, then we say that $G$ is intrinsically asymmetric.

We prove the following results:

Theorem 1. Let $H$ be a non-planar graph. Then there is a two-colouring $G$ of $H$ such that $G$ is intrinsically chiral, and if $H$ is 3-connected and is not $K_{3,3}$ or $K_{5}$, then $G$ is intrinsically asymmetric.

Theorem 2. Let $H$ be either $K_{3,3}$ or $K_{5}$. Then there does not exist a two-colouring of $H$ which is intrinsically asymmetric.

Motivated by the definition of the molecular symmetry group given by LonguetHiggins [4], Simon [6] introduced the concept of the topological symmetry group of a graph $G$ embedded in $S^{3}$. Simplifying his definition slightly we define the topological symmetry group of a graph $G$ embedded in $S^{3}$ to be $\operatorname{TSG}(G)=$ \{automorphisms of $G$ which are induced by a homeomorphism of $\left.S^{3}\right\}$. Observe that a graph $G$ is intrinsically asymmetric if and only if $\operatorname{TSG}(G)$ is trivial for each embedding of $G$ in $S^{3}$. We define a graph to be $\mathrm{n}$-connected if at least $\mathrm{n}$ vertices together with their incident edges must be removed in order to disconnect the graph or reduce it to a single vertex. Thus, we can interpret our main result to say that if $H$ is a non-planar 3connected graph which is not $K_{3,3}$ or $K_{5}$, then there is a two-colouring $G$ of $H$ such that for each embedding of $G$ in $S^{3}, T S G(G)$ is trivial.

Two topological tools that we will make use of are the Automorphism Theorem, which allows us to focus attention on finite order homeomorphisms of $S^{3}$, and Smith Theory, which characterizes the fixed point sets of such homeomorphisms. We state both of these results below.

Automorphism Theorem ([1]]). Let $G$ be a graph which is 3 -connected, and let $\phi$ be an automorphism of the vertices of $G$. Suppose that $G$ can be embedded in $S^{3}$ in such a way that $\phi$ is induced by a graph homeomorphism $h$ of $\left(S^{3}, G\right)$. Then there exists an embedding of $G$ in $S^{3}$ such that $\phi$ is induced by a finite order graph homeomorphism $f$ of $\left(S^{3}, G\right)$. Furthermore, $f$ is orientation reversing if and only if $h$ is orientation reversing.

Smith Theory ([7]). Let $f$ be a non-trivial finite order homeomorphism of $S^{3}$. If $f$ is orientation preserving then the fixed point set of $f$ is either the empty set or is a set which is homeomorphic to $S^{1}$. If $f$ is orientation reversing then the fixed point set of $f$ is either two points or a set which is homeomorphic to $S^{2}$.

We begin by proving the following corollary of the Automorphism Theorem.

Honeonorphism Lemma. Let $G$ be a 3-connected graph. Suppose that $G$ is embedded in $S^{3}$ in such a way that there exists a graph homeomorphism $h:\left(S^{3}, G\right) \rightarrow\left(S^{3}, G\right)$. If 
there exists a non-planar subgraph $T$ of $G$ all of whose vertices are fixed by $h$, then $h$ must fix all of the vertices of $G$.

Proof. Since $G$ is 3-connected, we can apply the Automorphism Theorem to get an embedding of $G$ in $S^{3}$ which has a finite order graph homeomorphism $f$ that induces the same automorphism as $h$ on the vertices of $G$. Note that $f$ fixes all of the vertices of $T$ and since $f$ has finite order, this means that $f$ fixes every point of $T$. By hypothesis, $T$ is non-planar, so it cannot be contained in $\varnothing, S^{0}, S^{1}$ or $S^{2}$. Therefore, by Smith Theory, $f$ must be the identity homeomorphism. Hence $h$ fixes all of the vertices of $G$. This concludes the proof of the Homeomorphism Lemma.

The graph $K_{5}$ consists of five vertices together with an edge between each pair of vertices. We prove another corollary of the Automorphism Theorem, which we shall also use in the proof of our main theorem.

$K_{5}$ Theorem. No matter how $K_{5}$ is embedded in $S^{3}$, there does not exist an orientation reversing graph homeomorphism of $\left(S^{3}, K_{5}\right)$, which either fixes every vertex of $K_{5}$, or induces an automorphism of $K_{5}$ of order 2 with only one fixed vertex.

Proof. Suppose that $K_{5}$ is embedded in such a way that there is an orientation reversing graph homeomorphism $h:\left(S^{3}, K_{5}\right) \rightarrow\left(S^{3}, K_{5}\right)$. Note that $K_{5}$ is 3 -connected. First we assume that $h$ induces the identity automorphism on the vertices of $K_{5}$. Then we can apply the Automorphism Theorem to get an embedding of $K_{5}$ in $S^{3}$ such that the identity automorphism on $K_{5}$ is induced by a finite order orientation reversing graph homeomorphism $f$ of $S^{3}$. Since $f$ is of finite order, this means that $f$ fixes every point of $K_{5}$. But this contradicts Smith Theory, since $K_{5}$ is non-planar and $f$ cannot be the identity since it is orientation reversing.

Now assume that $h$ induces an automorphism $\phi$ on the vertices of $K_{5}$ which is of order 2 and has only one fixed vertex. Let the vertices of $K_{5}$ be denoted by $1,2,3,4,5$. Without loss of generality, let $\phi=(12)(34)(5)$. Again, we apply the Automorphism Theorem to get an embedding of $K_{5}$ in $S^{3}$ such that $\phi$ is induced by a finite order orientation reversing graph homeomorphism $f$ of $\left(S^{3}, K_{5}\right)$. Note that $f$ fixes a point on the edge $\overline{12}$, a point on the edge $\overline{34}$ and vertex 5 , so $f$ fixes at least three points of $S^{3}$. Therefore, by Smith Theory, the fixed point set of $f$ is homeomorphic to a 2 -sphere $S$, which separates $S^{3}$ into two components $A$ and $B$. Without loss of generality, let vertices 1 and 3 be in $A$, then vertices 2 and 4 must be in $B$. Note that the edge $\overline{14}$ must intersect $S$. Thus a point on the edge $\overline{14}$ must be fixed by $f$, which implies that vertices 1 and 4 are interchanged by $f$. This is a contradiction, since $f$ interchanges vertices 1 and 2 . Therefore, $h$ cannot induce either the identity automorphism on the vertices of $K_{5}$, or an automorphism of order 2 with only one fixed vertex. This concludes the proof of the $K_{5}$ Theorem.

The graph $K_{3,3}$ consists of two disjoint sets of three vertices, with the edges being precisely those joining a vertex in one set to a vertex in the other set. Liang and Mislow [3] showed that the particular two-coloured embeddings of $K_{5}$ and $K_{3,3}$, illustrated in Figs. 1 and 2, respectively, are topologically chiral.

The abstract graph of a three-rung Möbius ladder $M_{3}$ is defined to be a simple closed curve $K$ containing six vertices called the loop, together with edges joining antipodal vertices on $K$ called rungs. Thus, a three-rung Möbius ladder $M_{3}$ is just a 


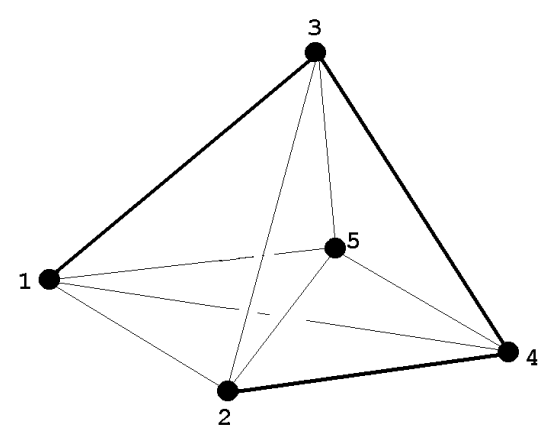

Fig. 1. A topologically chiral embedding of $K_{5}$.

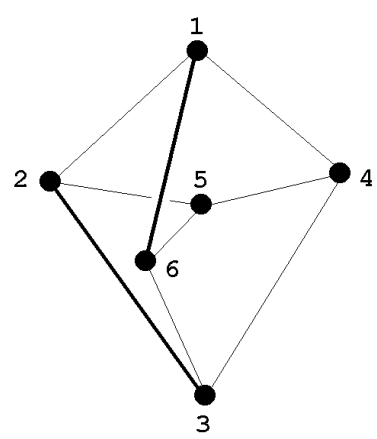

Fig. 2. A topologically chiral embedding of $K_{3,3}$.

$K_{3,3}$ with a specified simple closed curve containing all of the vertices. For example, the embedded $K_{3,3}$, shown in Fig. 2 , is an embedded three-rung Möbius ladder, where the rungs are the two black edges together with the edge $\overline{45}$, and the loop is $\overline{1256341}$.

We make the following observations about the two-colourings in Figs. 1 and 2. When $K_{5}$ is coloured as in Fig. 1, (12)(34)(5) is its only non-trivial automorphism, hence by the $K_{5}$ Theorem, this two-colouring is actually intrinsically chiral. Now consider the two-colouring of the $K_{3,3}$ in Fig. 2. The edge $\overline{45}$ in Fig. 2 is unique because it is the only white edge which is not adjacent to any black edge. Thus any automorphism of the two-coloured $M_{3}$ in Fig. 2 must take the white loop $\overline{1256341}$ to itself. It follows from the following theorem that this two-colouring of $K_{3,3}$ is intrinsically chiral.

Möbius Ladder Theorem ([2]). Let $M_{3}$ be a 3-rung Möbius ladder which is embedded in $S^{3}$ with loop $K$. Then there is no orientation reversing graph homeomorphism $h:\left(S^{3}, M_{3}\right) \rightarrow\left(S^{3}, M_{3}\right)$ such that $h\left(M_{3}\right)=M_{3}$ and $h(K)=K$.

Every non-planar graph $G$ must contain a subgraph $T$ which is homeomorphic to either $K_{3,3}$ or $K_{5}$. However, $T$ may not be graph homeomorphic to $K_{3,3}$ or $K_{5}$, since it might contain additional vertices of valence 2 . With this in mind we make the following definitions.

Definition. Let $T$ be a graph which is homeomorphic to $K_{3,3}$ or $K_{5}$. A simple path is a union of edges in $T$ which has no vertices of valence more than 2 in its interior. A path-edge is a simple path whose end-vertices have valence more than 2. 
The path-edges of $T$ correspond to the edges of $K_{3,3}$ or $K_{5}$.

In order to avoid confusing the coloured and uncoloured versions of a graph, we will use the letter $H$ to denote an uncoloured graph and the letter $G$ to denote the same graph after it has been two-coloured. We shall refer to $G$ as a two-colouring of $H$. Let $G$ be a two-coloured graph whose black subgraph is connected. A black tail $L$ is a black tree which can be disconnected from the rest of the black subgraph of $G$ by removing one vertex from $G$. If $p$ is a path, we shall let $|p|$ denote the number of edges contained in $p$.

Now we prove our main result.

Theorem 1. Let $H$ be a non-planar graph. Then there is a two-colouring $G$ of $H$ such that $G$ is intrinsically chiral, and if $H$ is 3-connected and is not $K_{3,3}$ or $K_{5}$, then $G$ is intrinsically asymmetric.

Proof. Since $H$ is non-planar, it must contain a subgraph which is homeomorphic to either $K_{3,3}$ or $K_{5}$, possibly with extra vertices. If $H$ contains a component which is $K_{3,3}$ or $K_{5}$, then we colour this particular $K_{3,3}$ or $K_{5}$ as in Fig. 2 or Fig. 1, respectively, and colour the rest of $H$ white. This two-colouring of $H$ is intrinsically chiral. So we shall assume throughout our proof that no component of $H$ is $K_{3,3}$ or $K_{5}$.

We begin by picking a subgraph $T$ of $H$ which is homeomorphic to either $K_{3,3}$ or $K_{5}$. The strategy of our proof will be to colour almost all of $H$ white except for a small black piece, whose edges are either contained in, or adjacent to $T$. This black piece will be chosen in such a way that it forces every automorphism of the two-colouring $G$ to fix each vertex of $T$. It will then follow from either the Möbius Ladder Theorem or the $K_{5}$ Theorem that $G$ is intrinsically chiral. Furthermore, if $H$ is 3-connected, then it will follow from the Homeomorphism Lemma, that $G$ is intrinsically asymmetric.

We divide the proof into two parts depending on whether the subgraph $T$ is $K_{3,3}$ or $K_{5}$. We will further divide each of these parts into cases and within each case we will specify how to colour $H$. First we suppose that $H$ contains a subgraph $T$ which is homeomorphic to $K_{3,3}$.

We label the vertices of $T$ corresponding to the vertices of $K_{3,3}$ by the numbers 1-6 in such a way that the vertices 1,3 and 5 are each connected to each of the vertices 2,4 and 6 . We shall divide the proof into two cases according to whether or not the path-edges of $T$ are all single edges.

Case I. Every path-edge of $T$ is a single edge.

In this case $T$ is the graph $K_{3,3}$. Since $K_{3,3}$ is not a component of $H$, there is some edge $e$ in $H-T$ which is connected to $T$. Without loss of generality, we can assume that one end-vertex of $e$ is vertex 6 . Let $m$ denote the other end-vertex of $e$.

First suppose that vertex $m$ is on $T$. Since vertex 6 is already connected to vertices 1,3 and $5, m$ can only be 2 or 4 . Without loss of generality, say vertex $m$ is vertex 2 . Then we colour $T \cup\{e\}$ as in Fig. 3 and we colour the rest of $H$ white. Let $G$ denote $H$ after it has been coloured and let $\mathscr{T}$ denote $T$ after it has been coloured. Let $h$ be an automorphism of $G$. Note that the simple closed curve $\overline{1261}$ is the only black simple closed curve in $G$, so $h(\overline{1261})=\overline{1261}$. Also, the two black tails $\overline{23}$ and $\overline{654}$ cannot be interchanged by $h$ since they have different lengths. Thus the vertices on 


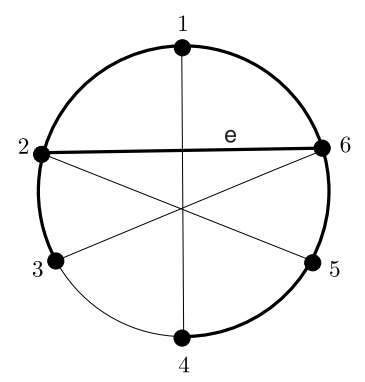

Fig. 3. Case I ( $m$ is on $T$ ).

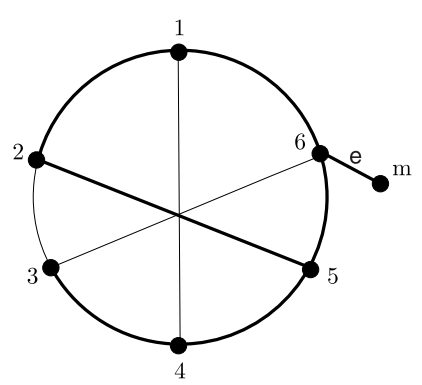

Fig. 4. Case $I$ ( $m$ is not on $T)$.

each tail are fixed by $h$. In particular, vertices 2 and 6 are both fixed by $h$. Hence $h(\overline{\mathbf{2 6}})=\overline{\mathbf{2 6}}$ since $\overline{\mathbf{2 6}}$ is a single edge. It follows that $h$ fixes every vertex of $\mathscr{T}$.

Now suppose that vertex $m$ is not on $T$. Then we colour $T \bigcup\{e\}$ as in Fig. 4 and colour the rest of $H$ white. The argument is then analogous to the above paragraph.

Case II. T contains at least one path-edge which is not a single edge.

We subdivide this case according to how many mutually non-adjacent path-edges there are in $T$ which are single edges.

Subcase 1. T contains at least three mutually non-adjacent path-edges which are each single edges.

Without loss of generality we can assume that $\overline{14}, \overline{25}$ and $\overline{36}$ are single edges, and $\overline{16}$ is not a single edge. Let $x$ be the vertex on the path-edge $\overline{16}$ such that $\overline{6 x}$ is a single edge. If $\overline{1 x}$ is not a single edge, let $y$ be the vertex on the path-edge $\overline{16}$ such that $\overline{x y}$ is a single edge. We mark each path-edge which is known to be a single edge by a letter ' $S$ '. If vertex $y$ exists, then we colour $T$ as in Fig. 5 and colour the rest of $H$ white. If vertex $y$ does not exist, then we modify the colouring in Fig. 5 by colouring the edge $\overline{1 x}$ white. The argument is now analogous to that of Case I.

Subcase 2. T contains two non-adjacent path-edges which are each single edges, but $T$ does not contain three mutually non-adjacent path-edges which are each single edges.

Without loss of generality we assume that the path-edges $\overline{25}$ and $\overline{34}$ are single edges, while $\overline{\mathbf{1 6}}$ is not a single edge. By the hypothesis of this case, since $\overline{25}$ is a single edge, at least one of $\overline{14}$ and $\overline{36}$ is not a single edge. So without loss of generality we assume that $\overline{14}$ is not a single edge. If $\overline{36}$ is not a single edge let $x$ be the vertex on $\overline{36}$ such that $\overline{x 6}$ is a single edge, otherwise let $x=3$. Since $\overline{16}$ is not a single edge, we 


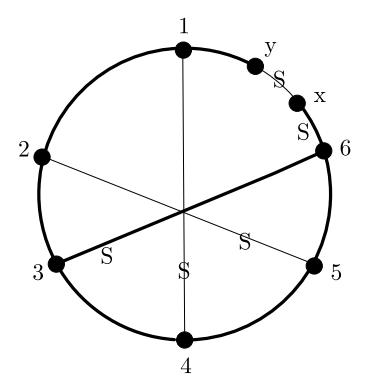

Fig. 5. Case II, subcase 1.

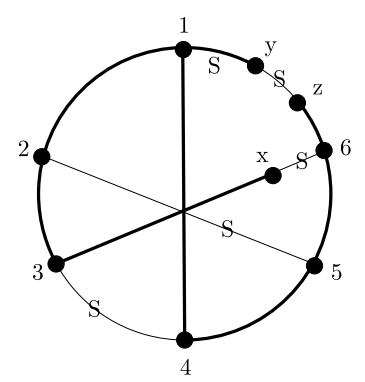

Fig. 6. Case II, subcase $2(|\overline{123 x}| \neq|\overline{1456 z}|)$.

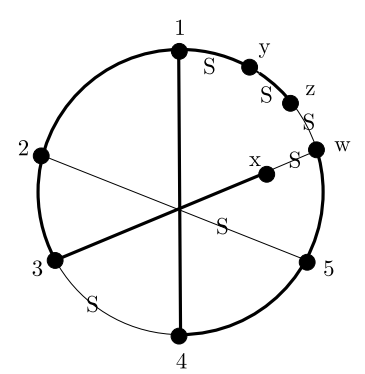

Fig. 7. Case II, subcase $2(|\overline{123 x}|=|\overline{1456 z}|)$.

let $y$ denote the vertex on $\overline{16}$ such that $\overline{1 y}$ is a single edge. If $\overline{y 6}$ is not a single edge we let $z$ denote the vertex on $\overline{y 6}$ such that $\overline{y z}$ is a single edge, otherwise we let $z=6$. Now if $|\overline{123 x}| \neq|\overline{1456 z}|$ then we colour $T$ as in Fig. 6 and colour the rest of $H$ white.

Suppose that $|\overline{123 x}|=|\overline{1456 z}|$. Let $w$ be a vertex on $\overline{z 5}$ such that $\overline{z w}$ is a single edge. Note that $w$ might be 6 , or if $z=6$ then $w$ might be 5 . Then $|\overline{1456 z}|=|\overline{145 w}|+1$, since $\overline{z w}$ is a single edge. So $|\overline{123 x}|>|\overline{145 w}|$. Also $|\overline{1 y z}|=2$, and $|\overline{145 w}| \geqslant 3$, since $\overline{14}$ is not a single edge. Now we colour $T$ as in Fig. 7 and colour the rest of $H$ white.

Let $G$ denote the two-colouring of $H$ indicated in Fig. 6 or Fig. 7, and let $\mathscr{T}$ denote $T$ after it has been coloured. The black subgraph of $G$ is a tree with three branches, all of different lengths. Every vertex of $\mathscr{T}$ is contained in this black tree. Let $h$ denote an automorphism of $G$. Then $h$ fixes every vertex of this black tree, and thus $h$ fixes every vertex of $\mathscr{T}$.

Subcase 3. $T$ does not contain a pair of non-adjacent path-edges which are single edges. 


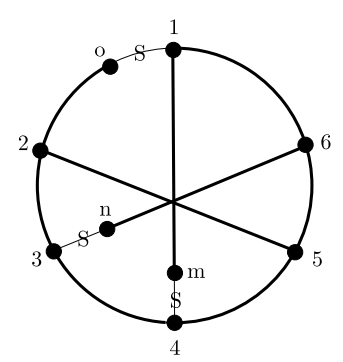

Fig. 8. Case II, subcase 3 .

Without loss of generality, assume that the vertex labels have been chosen so that path-edge $\overline{25}$ has minimal length among all path-edges and $|\overline{63}| \leqslant|\overline{14}|$. By the hypothesis of this subcase, neither $\overline{63}$ nor $\overline{14}$ is a single edge and at least one of $\overline{12}$ or $\overline{45}$ is not a single edge. We shall assume without loss of generality that $\overline{12}$ is not a single edge.

Let vertices $o, n$ and $m$ on $\overline{12}, \overline{63}$ and $\overline{14}$, respectively, denote the vertices such that $\overline{1 o}, \overline{3 n}$ and $\overline{4 m}$ are single edges. We colour $T$ as in Fig. 8 and colour the rest of $H$ white. We shall let $G$ denote $H$ after it has been coloured and let $\mathscr{T}$ denote $T$ after it has been coloured. Let $h$ be an automorphism of $G$. First observe that the black subgraph of $G$ consists of a simple closed curve $\overline{23452}$ with two disjoint tails $\overline{2 o}$ and $\overline{56 n} \cup \overline{61 m}$ which are not homeomorphic to each other. Thus $h$ fixes each of the vertices on $\overline{2 o}$ and $\overline{56}$. Since $|\overline{63}| \leqslant|\overline{14}|$ by hypothesis, we know that $|\overline{6 n}|<|\overline{61 m}|$. Thus $h$ cannot interchange $\overline{6 n}$ and $\overline{61 m}$. So $h$ fixes all of the vertices on $\overline{6 n}$ and $\overline{61 m}$. Since $\overline{25}$ has minimal length among all path-edges, we have $|\overline{25}|<|\overline{2345}|$. So, $h$ cannot exchange $\overline{25}$ and $\overline{2345}$. Therefore $h(\overline{25})=\overline{25}$ and $h(\overline{2345})=\overline{2345}$. Thus every vertex on $\mathscr{T}$ is fixed by $h$.

From these cases, it follows that if a graph $H$ does not have a component which is $K_{3,3}$ but contains a subgraph $T$ homeomorphic to $K_{3,3}$, then a two-colouring $G$ can be chosen such that any automorphism of $G$ induces the identity automorphism on $T$. It follows from the Möbius Ladder Theorem that $G$ is intrinsically chiral. If in addition, $H$ is 3 -connected, then by the Homeomorphism Lemma, $G$ is intrinsically asymmetric.

Now we suppose that $H$ contains a subgraph $T$ which is homeomorphic to $K_{5}$. We label the vertices of $T$ which correspond to those of $K_{5}$ by the numbers $1-5$. In order to help us talk about the parts of a two-coloured graph which are black, we will make the following definitions.

Definition. Let $G$ be a two-coloured graph whose black subgraph is connected. A black edge is non-trivial if it is not part of a black tail. The non-trivial black valence of a vertex $v \in G$ is the number of non-trivial black edges which are incident to $v$. A major black neighbour of $v$ is a vertex which has black valence more than 2 and is connected to $v$ by a simple black path.

Case I. There exists a path in $H-T$ from a vertex in the interior of a path-edge of $T$ to one of the vertices numbered 1-5.

Without loss of generality, assume that the vertex labels have been chosen so that 
Table 1. Case I, subcase 1

\begin{tabular}{ccc}
\hline Vertex & Non-trivial black valence of MBN & Number of MBN with a black tail \\
\hline$m$ & 3 & 1 \\
1 & $3,4,5$ & 0 \\
3 & $4,4,5$ & 0 \\
$x$ & $3,4,5$ & 1 \\
2 & $3,3,4,5$ & 1 \\
5 & $3,3,4,5$ & 0 \\
4 & $3,3,3,4,4$ & 1 \\
\hline
\end{tabular}

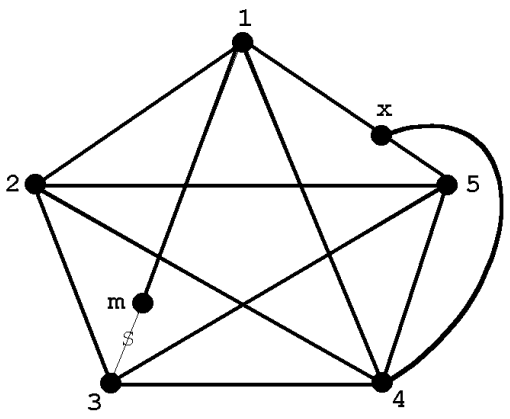

Fig. 9. Case I, subcase 1.

there is a path in $H-T$ from a vertex in the interior of the path-edge $\overline{15}$ to vertex 4. We now divide this case into the following two subcases.

Subcase 1. At least one of the path-edges $\overline{12}, \overline{13}, \overline{52}$ and $\overline{53}$ is not a single edge.

Note that the positions of the above four path-edges are identical relative to the path $\overline{4 x}$. So, without loss of generality, we can assume that the vertex labels have been chosen so that the path-edge $\overline{13}$ is not a single edge. Let vertex $m$ be the vertex on $\overline{13}$ such that $\overline{3 m}$ is a single edge. We colour $T$ as in Fig. 9 and colour the rest of $H$ white.

We shall let $G$ denote $H$ after it has been coloured and let $\mathscr{T}$ denote $T$ after it has been coloured. Let $h$ be an automorphism of $G$. Table 1 lists vertices numbered $1-5$, together with vertices $m$ and $x$, and enumerates the non-trivial black valence of each of their major black neighbours (MBN) and the number of their MBN which has a black tail. All the information in Table 1 will be preserved by the automorphism $h$.

Note that every vertex in Table 1 has some unique property that will be preserved by $h$. Thus, the vertices in Table 1 are all fixed by $h$. It follows that every vertex in $\mathscr{T}$ is fixed by $h$.

Subcase 2 . The path-edges $\overline{12}, \overline{13}, \overline{52}$ and $\overline{53}$ are all single edges.

If the path-edge $\overline{\mathbf{1 4}}$ is not a single edge, we let vertex $m$ be the vertex on $\overline{\mathbf{1 4}}$ such that $\overline{4 m}$ is a single edge. Similarly if the path-edge $\overline{34}$ is not a single edge, we let vertex $n$ be the vertex on $\overline{34}$ such that $\overline{3 n}$ is a single edge. If vertices $m$ and $n$ exist, then colour $T$ as in Fig. 10 and colour the rest of $H$ white. If vertex $m$ does not exist, modify the colouring of Fig. 10 by colouring $\overline{\mathbf{1 4}}$ white, and if vertex $n$ does not exist, modify the colouring of Fig. 10 by colouring $\overline{34}$ white. 


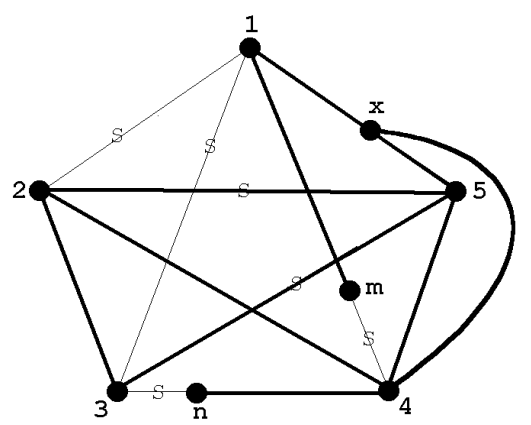

Fig. 10. Case I, subcase 2 .

Table 2. Case I, subcase 2

\begin{tabular}{ccc}
\hline Vertex & Non-trivial black valence of MBN & Number of black tails of vertex \\
\hline 2 & 3,4 & 0 \\
$x$ & 3,4 & 1 \\
4 & $2,3,4$ & 0 or 1 \\
5 & $2,3,3$ & 0 \\
\hline
\end{tabular}

We shall let $G$ denote $H$ after it has been coloured and let $\mathscr{T}$ denote $T$ after it has been coloured. Let $h$ be an automorphism of $G$. Table 2 lists all of the vertices in $\mathscr{T}$ with black valence at least 3 , and enumerates the non-trivial black valence of each of their MBN and the number of black tails attached to each vertex.

Note that each of the vertices in Table 2 is fixed by $h$ since each has some unique property that will be preserved by $h$. Consequently vertex 1 is fixed and hence vertex $m$ is fixed (if it exists). Also, if vertex $n$ exists, then it is fixed since vertex 4 is fixed. Since $\overline{25}$ is a single edge and the paths $\overline{25}$ and $\overline{235}$ are the only simple black paths between vertices 2 and 5 . It follows that $h$ maps $\overline{235}$ to itself and vertex 3 is fixed. Thus, $h$ induces the identity automorphism on the vertices of $\mathscr{T}$.

Case II. There does not exist any path in $H-T$ from a vertex in the interior of a path-edge of $T$ to one of the vertices numbered 1-5.

Subcase 1. There exists some path-edge of $T$ which is not a single edge.

Without loss of generality, we can assume that the vertex labels have been chosen so that path-edge $\overline{12}$ is not a single edge. Let vertex $m$ be the vertex on $\overline{12}$ such that $\overline{2 m}$ is a single edge. If either $\overline{23}$ or $\overline{34}$ is not a single edge, we let vertices $n$ or $o$ on $\overline{23}$ or $\overline{34}$, respectively, denote vertices such that $\overline{3 n}$ or $\overline{4 o}$ is a single edge. If vertices $n$ and $o$ exist, then colour $T$ as in Fig. 11 and colour the rest of $H$ white. If either vertex $n$ or $o$ does not exist, modify the colouring of Fig. 11 by colouring the edge $\overline{23}$ or $\overline{34}$ (respectively) white.

We shall let $G$ denote $H$ after it has been coloured and let $\mathscr{T}$ denote $T$ after it has been coloured. Let $h$ be an automorphism of $G$. First note that vertex 5 must be fixed by $h$ since it is the only vertex of $G$ with non-trivial black valence 4 . Similarly, vertex 1 is fixed by $h$ since it is the only vertex with non-trivial black valence 3 and a black tail, and vertex 4 is fixed by $h$ since it is the only vertex with non-trivial black valence 3 and no black tail. Note that vertex 3 cannot be mapped to vertex 2 , 


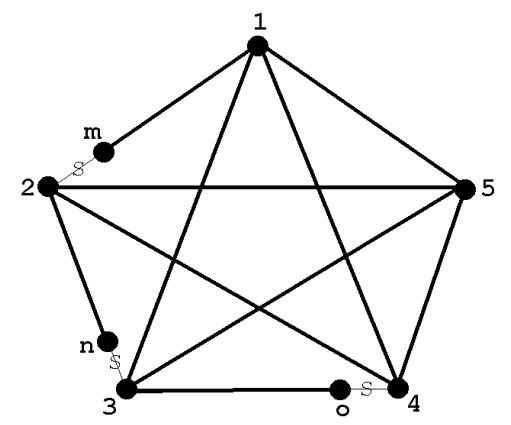

Fig. 11. Case II, subcase 1.

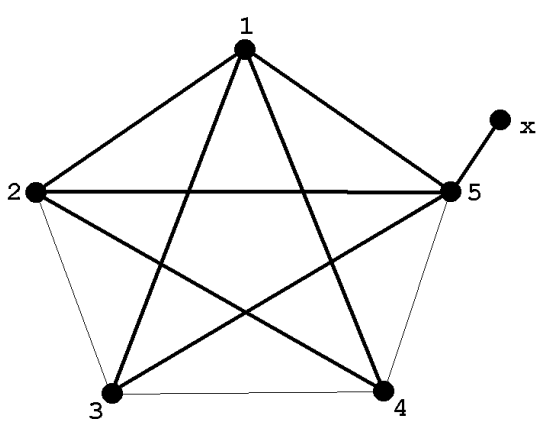

Fig. 12. Case II, subcase 2.

since vertex 3 is connected to vertex 1 by a simple black path while vertex 2 is not. Suppose that vertex 3 were mapped to a vertex in the interior of $\overline{15}$. Then vertex $o$ would not exist and $\overline{34}$ would be a single white edge. Since $h(4)=4$, there must be a single white edge from vertex 4 to a vertex in the interior of $\overline{15}$. Such an edge must be contained in $H-T$. This is contrary to the hypothesis of Case II. Thus we must have $h(\overline{\mathbf{1 3 5}})=\overline{135}$. So vertex 3 is fixed and vertex $o$ is fixed if it exists. By a similar argument, vertex 2 is also fixed by $h$, and vertex $n$ is fixed if it exists. Therefore every vertex of $\mathscr{T}$ is fixed by $h$.

Subcase 2. Every path-edge of $T$ is a single edge.

Since $T$ is just $K_{5}$, it follows from the beginning of our proof that $T$ is not a component of $H$. Hence, there must be some vertex in $H-T$ which is connected to $T$ by a single edge. Without loss of generality, let $x$ be a vertex in $H-T$ which is connected to vertex 5 by a single edge. We colour $T \bigcup\{\overline{5 x}\}$ as in Fig. 12 and colour the rest of $H$ white.

We shall let $G$ denote $H$ after it has been coloured and let $\mathscr{T}$ denote $T$ after it has been coloured. Let $h$ be an automorphism of $G$. By considering non-trivial black valence together with the existence of a black tail, it is easy to see that $h$ fixes vertices 1,2 and 5 . Also the single edge $\overline{35}$ is black while the single edge $\overline{45}$ is white. Thus, $h$ induces the identity automorphism on the vertices of $\mathscr{T}$.

In all of the above cases it follows that if a graph $H$ does not have a component which is $K_{5}$ but contains a subgraph $T$ homeomorphic to $K_{5}$, then a two-colouring $G$ 


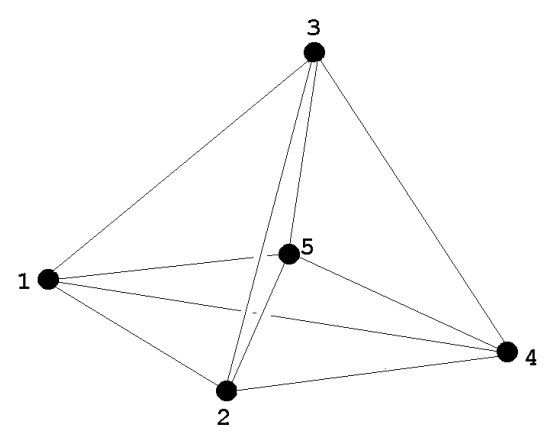

Fig. 13. An embedding of $K_{5}$.

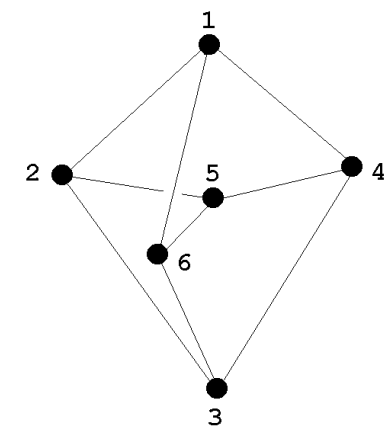

Fig. 14. An embedding of $K_{3,3}$.

can be chosen such that any automorphism of $G$ induces the identity automorphism on $T$. Then, by the $K_{5}$ Theorem, $G$ is intrinsically chiral. Furthermore, if $H$ is 3connected, then by the Homeomorphism Lemma, $G$ is intrinsically asymmetric. This concludes the proof of Theorem 1 .

We show below that the hypothesis of Theorem 1 that $H$ was neither $K_{3,3}$ nor $K_{5}$ was strictly necessary to guarantee the existence of an intrinsically asymmetric two-colouring.

Theorem 2. Let $H$ be either $K_{3,3}$ or $K_{5}$, then there does not exist a two-colouring of $H$ which is intrinsically asymmetric.

Proof. We will show that for every two-colouring $G$ of the embeddings illustrated in Figs. 13 and 14 there is a graph homeomorphism of $\left(S^{3}, G\right)$ which induces a nontrivial automorphism of $G$. For any two-colouring $G$ of $H$, we let $T$ denote its black subgraph. Note that $K_{5}$ contains precisely ten edges and $K_{3,3}$ contains precisely nine edges; so, without loss of generality, we can assume that $T$ contains at most five edges since otherwise we could consider the white subgraphs of $G$ instead of the black subgraphs. We can also assume that $T$ has at least one edge, since otherwise $G$ is entirely white and obviously has a non-trivial automorphism induced by a homeomorphism of $S^{3}$ for the embeddings illustrated in Figs. 13 and 14. Tables 3 and 4 list all possible subgraphs of $K_{5}$ and $K_{3,3}$ which have the given number of edges (indicated at the top of each column). We do not distinguish between different numberings of the vertices. It follows by inspection that every $T$ in Table 3 has a 


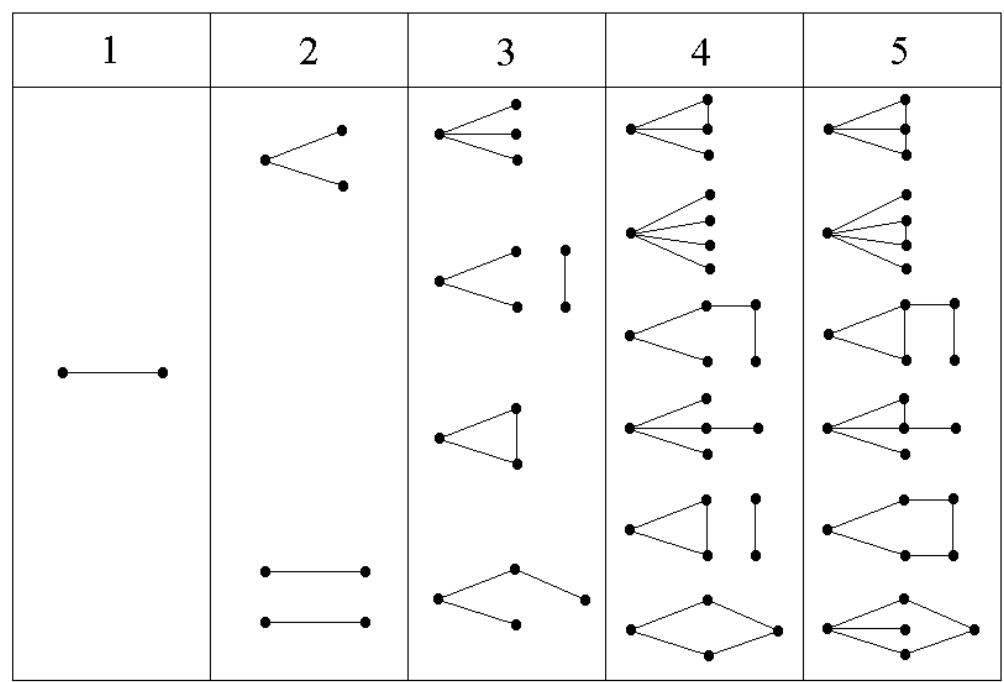

Table 3. Black subgraphs of $K_{5}$ containing up to five edges

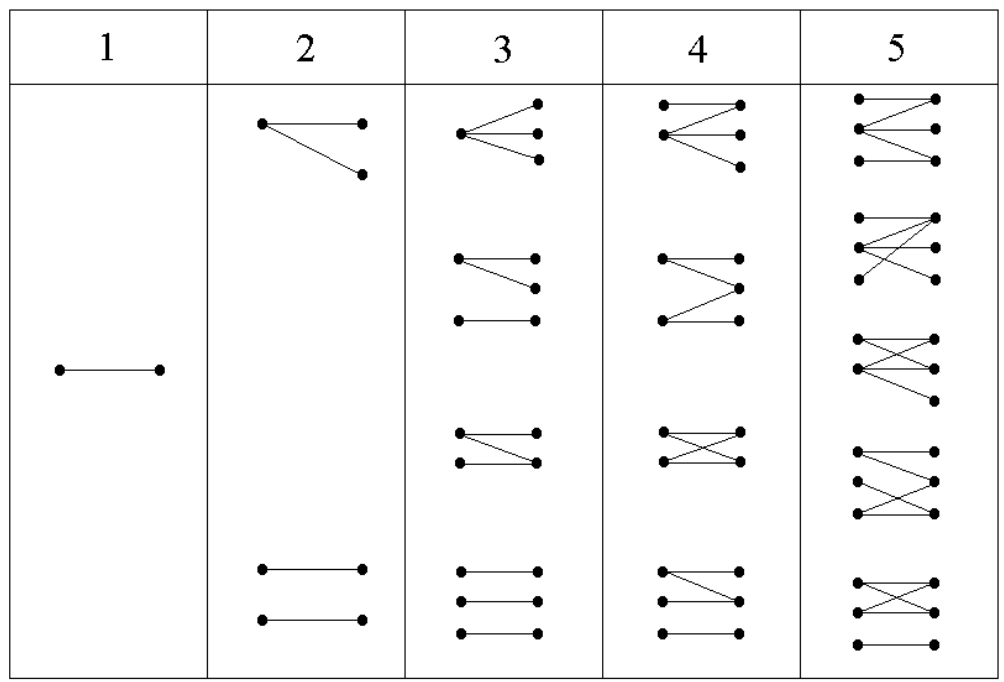

Table 4. Black subgraphs of $K_{3,3}$ containing up to five edges

non-trivial automorphism. If $H$ is $K_{5}$, we extend this non-trivial automorphism of $T$ to $K_{5}$ by fixing those vertices in $H-T$. This extension is an automorphism of $K_{5}$ because each vertex in $K_{5}$ is connected to all other vertices. If $H$ is $K_{3,3}$, then there is a unique way to partition $H$ into two sets $A$ and $B$ which are each mutually non-adjacent. Hence, every automorphism of $K_{3,3}$ either leaves the sets $A$ and $B$ invariant or interchanges the sets $A$ and $B$. We observe that every $T$ in Table 4 has a non-trivial automorphism which either takes $A \cap T$ to $A \cap T$ and $B \cap T$ to $B \cap T$, or exchanges the sets $A \cap T$ and $B \cap T$. We extend a non-trivial automorphism of $T$ of the first type to an automorphism of $K_{3,3}$ by fixing each of the vertices in $H-T$. We extend a non-trivial automorphism of $T$ of the second type to an automorphism of $K_{3,3}$ by interchanging the vertices in $(H-T) \cap A$ with the vertices in $(H-T) \cap B$. 


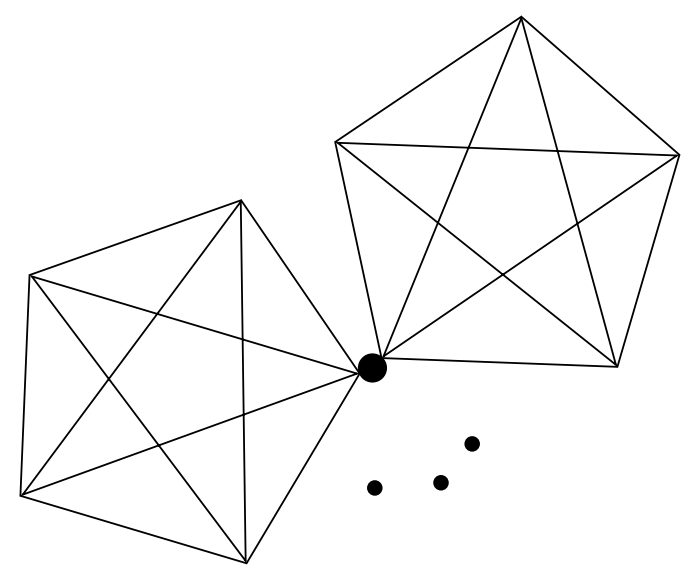

Fig. 15. $\left(2^{10}+1\right)$ copies of $K_{5}$ sharing one common vertex.

Given any two-colouring $G$ of the abstract graph of $K_{5}$ or $K_{3,3}$, we embed $G$ as is shown in Figs. 13 or 14 respectively. By our above argument, $G$ has a non-trivial automorphism $\phi$. It follows from [5] that every automorphism $\phi$ of $K_{5}$ or $K_{3,3}$, is induced by a homeomorphism of $S^{3}$ for the embeddings shown in Fig. 13 or 14. Thus we have a homeomorphism of $S^{3}$ which induces a non-trivial automorphism $\phi$ of $G$. Hence, $H$ cannot be two-coloured to be intrinsically asymmetric. This concludes the proof of Theorem 2 .

Finally, we note that if $H$ is not 3-connected, then there does not necessarily exist an intrinsically asymmetric two-colouring of $H$. For example, consider the graph $H$ in Fig. 15, consisting of $\left(2^{10}+1\right)$ copies of $K_{5}$ which share one common vertex, but are otherwise disjoint. Since there are at most $2^{10}$ possible two-colourings of a $K_{5}$ graph, no matter how we two-colour $H$, there will exist at least one pair of $K_{5}$ graphs in $H$ with identical colourings. So, no matter how we two-colour $H$, we can embed $H$ in $S^{3}$ in such a way that there exists a graph homeomorphism $h:\left(S^{3}, H\right) \rightarrow\left(S^{3}, H\right)$, which interchanges two $K_{5}$ graphs with identical colourings and fixes the rest of $H$ pointwise. Thus $H$ has no intrinsically asymmetric two-colouring.

Similarly, we can create a 2 -connected graph which is not 3 -connected consisting of $\left(2^{10}+1\right)$ copies of $K_{5}$ which all share a common edge. By an analogous argument, there does not exist a two-colouring of this graph which is intrinsically asymmetric.

\section{REFERENCES}

[1] E. Flapan. Rigidity of graph symmetries in the 3-sphere. J. Knot Theory Ramifications 4 (1995), 373-388

[2] E. Flapan. Symmetries of Möbius ladders. Math. Ann. 283 (1989), 271-283.

[3] C. Liang and K. Mislow. Topological chirality of minimally coloured Kuratowski graphs. Croatica Chemica Acta 70 (1996), 735-744.

[4] H. C. Longuet-Higgins. The symmetry groups of non-rigid molecules. Molec. Phys. 6 (1963), $445-460$.

[5] N. Robertson, P. Seymour and R. Thomas. Sachs' linkless embedding conjecture. J. Combin. Theory Ser. B, 64 (1995), 185-227.

[6] J. Simon. A topological approach to the stereochemistry of nonrigid molecules. In Graph theory and topology in chemistry (eds. R. B. King and D. H. Rouvray), (Elsevier, 1987), pp. 43-75.

[7] P. A. Sмiтh. Transformations of finite period II. Ann. Math. 40 (1939), 690-711. 